\title{
ESTUDIO SOBRE LA DINÁMICA TEMPORAL DE MATERIAL PARTICULADO PM 10 EMITIDO EN COCHABAMBA, BOLIVIA
}

\author{
Giovanni Angelo SALINI CALDERÓN ${ }^{1 *}$ y Evelin Jhovana MEDINA MITMA²
}

${ }^{1}$ Universidad Católica de la Santísima Concepción. Alonso de Rivera 2850, Concepción, Chile

${ }^{2}$ Universidad Mayor de San Simón. Calle Sucre y Parque La Torre, Cochabamba, Bolivia

*Autor para correspondencia: gsalini@ucsc.cl

(Recibido noviembre 2015; aceptado marzo 2016)

Palabras clave: contaminación ambiental, dinámica no lineal, calidad del aire, series temporales no lineales, caos

\section{RESUMEN}

En este documento se presenta un estudio de series temporales de $\mathrm{PM}_{10}$ que muestran la mala calidad del aire en Cochabamba, mediante parámetros estadísticos usados en estudios sobre dinámica no lineal. El promedio diario de $\mathrm{PM}_{10}$ sigue patrones similares al de grandes ciudades que poseen altos índices de contaminación ambiental. Uno de los parámetros resultó del mismo orden y característica que los presentados en trabajos similares sobre el estudio de caoticidad en variables de contaminación como ozono, $\mathrm{PM}_{2.5}$, y CO, demostrando el origen caótico de estos datos. Nuestros resultados contribuyen a la literatura puesto que introducen un análisis metodológico más detallado de la naturaleza no lineal del contaminante $\mathrm{PM}_{10}$.

Key words: air pollution, nonlinear dynamics, air quality, nonlinear time series, chaos

\begin{abstract}
This paper presents a study of $\mathrm{PM}_{10}$ time series showing the poor air quality at Cochabamba city through statistical tools used in studies on nonlinear dynamics. The daily average of $\mathrm{PM}_{10}$ follows patterns similar to large cities with high of environmental pollution patterns. One of the parameters resulted from the same order and characteristic as those presented in similar studies on the chaos in pollution variables such as ozone, $\mathrm{PM}_{2.5}, \mathrm{CO}$, demonstrating the chaotic origin of these data. Our results contribute to the literature since they introduce a more detailed methodological analysis of the nonlinear nature of $\mathrm{PM}_{10}$ concentration.
\end{abstract}

\section{INTRODUCCIÓN}

El estudio de la contaminación atmosférica ha adquirido relevancia a nivel mundial (Molina y Molina 2004), consistiendo en la liberación de elementos nocivos en grandes cantidades que producen daño al ser humano y a su entorno. Esta contaminación resulta de cierta condición atmosférica en que sustancias nocivas alcanzan concentraciones superiores al nivel considerado normal (OMS 2006). Las partículas totales del aire en suspensión (PTS) son sólidas o líquidas e incluyen contaminantes primarios -polvo, monóxido 
de carbono, material particulado-, y contaminantes secundarios -aquellos que resultan de la interacción de varios contaminante primarios. De especial interés son los aerosoles atmosféricos, (o material particulado-PM) constituido por polvo del ambiente. Entre éstos se encuentra el PM de diámetro igual o menor a $10 \mathrm{~mm}\left(\mathrm{PM}_{10}\right)$, denominada fracción gruesa y $\mathrm{PM}$ de diámetro igual o menor a $2.5 \mathrm{~mm}\left(\mathrm{PM}_{2.5}\right)$ denominada fracción fina. La contaminación del aire es un grave problema para la salud humana pues en el mundo 1.4 de 6 millones de muertes anuales son atribuibles a dicha contaminación (OMS 2006). Muchos estudios muestran el daño que la contaminación provoca en la salud humana (Ilabaca et al. 1999), en la fauna y en la vegetación, existiendo especial interés en la investigación de los contaminantes orgánicos atmosféricos, debido a que altas concentraciones en PM de estas sustancias poseen propiedades carcinogénicas o mutagénicas, pudiendo ocasionar trastornos a la salud de la población (Raabe 1999, Schwartz et al. 2002). Un factor importante sobre el efecto en la salud es el tamaño de las partículas debido al grado de penetración y permanencia que ellas tienen en el sistema respiratorio humano. Así, partículas cuyo diámetro sea mayor a $5 \mathrm{~mm}$ se depositan en las vías aéreas superiores, en la tráquea y los bronquios, y aquellas cuyo diámetro es menor a dicho valor tienen mayor probabilidad de depositarse en los bronquiolos y alveolos (Peters y Dockery 2005), e inclusive la fracción fina puede llegar hasta la sangre (Cifuentes et al. 2000). Se ha establecido que por cada $50 \mu \mathrm{g} / \mathrm{m}^{3}$ de aumento de las concentraciones de $24 \mathrm{~h}$ de $\mathrm{PM}_{10}$ -en promedio- se verifica un incremento de $3 \%$ en la mortalidad (Morales 2006). El desarrollo urbano, la creciente industrialización así como el incremento del parque automotor lleva consigo al aumento de la contaminación ambiental, fenómeno que está siendo observado en todo el mundo (Molina y Molina 2004).

\section{Calidad del aire en Bolivia}

Bolivia cuenta con una regulación ambiental, denominada Ley General del Medioambiente 1333, promulgada en el año 1992 (Reglamentación Ley del Medioambiente $\mathrm{N}^{\mathrm{o}} 1333,1992$ ), en que se norma al material particulado de diámetro menor o igual a $10 \mathrm{~mm}$, entre otros contaminantes. Según esta ley la concentración límite para las partículas de diámetro menores a $10 \mathrm{~mm}\left(\mathrm{PM}_{10}\right)$ es $150 \mu \mathrm{g} / \mathrm{m}^{3}$ para $24 \mathrm{~h}$ y
$50 \mu \mathrm{g} / \mathrm{m}^{3}$ para la media geométrica anual ${ }^{1}$. Según un estudio de calidad del aire, elaborado por el Ministerio del Medio Ambiente y Agua (MMAyA) a través de la Red de Monitoreo de la Calidad del Aire (Red MoniCA) y el programa Aire Limpio de Swisscontact (Bacopé-Moscoso 2003), la ciudad con mayor problema de contaminación es Cochabamba causado por la acción humana, el aumento del parque vehicular y la morfología de la ciudad (Vargas y Luján 2006, Melgarejo et al. 2010). Además, es una de las 10 ciudades más contaminadas de América Latina (MMAyA 2010) y presenta características geográficas similares a Santiago de Chile, pues se encuentra ubicada entre valles y cerros con poco viento y lluvia; situación que es crítica en época invernal. Dicho estudio asevera que la contaminación en Cochabamba es provocada por material particulado grueso, fino y ultrafino, proveniente de quemas no autorizadas, fuentes móviles y fábricas ladrilleras (Vargas y Luján 2006). La dificultad en diseñar estrategias para reducir los niveles de contaminación se debe a la naturaleza altamente no lineal de las partículas atmosféricas y a la complejidad de las interacciones físicas y químicas que se dan en la atmósfera (Salini y Pérez 2015). La temporada de lluvias en Bolivia es entre los meses de noviembre a mayo (invierno altiplánico). En época invernal -entre junio y septiembre-, Cochabamba tiene momentos donde se registran altas concentraciones de contaminantes y una lenta disipación de ellos debido a las bajas temperaturas, la escasa lluvia, la falta de viento y escases de áreas verdes, así como a la altura de esta ciudad (Díaz y Páez 2006). Estos factores, más la disminución de la presión del aire, provocan el limitado ingreso de oxígeno al motor, causando que un vehículo contamine más en esta ciudad que en una que esté en zonas de más baja altura. Al estar en un valle atrapado entre serranías y cordilleras permite que se forme una capa de inversión térmica (capas de aire tibio entre otras frías) y provoca que los contaminantes tengan un periodo de vida más largo, impidiendo su dispersión adecuada. Según el inventario de emisiones generado entre los años 2008 y 2009 (Pareja et al. 2011), 92 \% de la contaminación es debido al parque automotor. Los principales gases tóxicos son dióxido de nitrógeno $\left(\mathrm{NO}_{2}\right)$, ozono $\left(\mathrm{O}_{3}\right)$ y material particulado $\left(\mathrm{PM}_{10}\right)$, presentando tendencia al aumento debido a las fuentes fijas y móviles, y al

${ }^{1}$ Los valores de concentración están referidos a concentraciones normales de presión y temperatura, considerándose para: Presión $=1 \mathrm{~atm}$ y Temperatura $=298 \mathrm{~K}\left(25^{\circ} \mathrm{C}\right)$. Los valores de este cuadro admiten una variación de hasta $+10 \%$. 
incremento poblacional. Según datos de las estaciones de monitoreo que miden PM y gases, la fuente que emitió mayor cantidad de contaminantes en la ciudad $(70 \%$ ) fue el transporte público (Vargas y Luján 2006, Red MoniCA 2010).

\section{Métodos no lineales en series temporales}

En el análisis del comportamiento de series temporales de PM que presentan comportamiento no lineal se emplean herramientas de sistemas dinámicos, puesto que pequeñas causas producen grandes efectos en valores futuros (Lorenz 1963), siendo ésta la esencia de la no linealidad. Pérez y Reyes (2001) mostraron una metodología para determinar la dimensión de encajamiento y tiempo de rezago óptimos para series de concentraciones horaria de $\mathrm{PM}_{10}$ en Santiago de Chile. El tiempo de rezago se determina mediante la función AMI (Average Mutual Information o Información Mutua Promedio, Fraser y Swinney 1986). Esta función posee un mínimo en cierto tiempo $\tau$. Este primer mínimo hallado se considera un estimador para llevar a cabo la reconstrucción del atractor en el espacio de las fases (Abarbanel 1996, Salini y Pérez 2006). Con esto se logra encontrar la separación temporal óptima de datos necesarios para capturar la dinámica interna, a través de los rasgos más relevantes de las series semejantes (en cuanto a condiciones de borde), relativos a la concentración de $\mathrm{PM}_{10}$ en la atmósfera (Salini y Pérez 2006 y 2015). Por su parte, la dimensión de encajamiento $d_{e}$ corresponde a la longitud de una secuencia de observaciones en una serie temporal. Según Takens (1981), basta con conocer una de las componentes de las trayectorias para reconstruir el atractor del sistema original (serie unidimensional $\left\{\mathrm{x}_{\mathrm{k}}\right\}$ ) pudiéndose construir una colección de vectores dado por

$\mathrm{y}(\mathrm{k})=\left(\mathrm{s}(\mathrm{k}), \mathrm{s}(\mathrm{k}+\tau), \ldots, \mathrm{s}\left(\mathrm{k}+\left(d_{e}-1\right) \tau\right)\right)$,

El método de falsos vecinos más cercanos (Función FNN o False Nearest Neighbors) permite determinar la dimensión de encajamiento $d_{e}(\tau)$, correspondiendo a la mínima cantidad de variables dinámicas necesaria para modelar al sistema (Salini y Pérez 2006).

Según Lorenz (1963) la no linealidad es inherente a los sistemas atmosféricos. Sivakumar destaca la existencia del comportamiento no lineal en el índice de calidad del aire (Sivakumar et al. 2007) en series temporales de ozono $\left(\mathrm{O}_{3}\right)$. Raga y Le Moine (1996) aplicaron herramientas de dinámica no lineal en datos de calidad del aire como $\mathrm{NO}, \mathrm{CO}, \mathrm{SO}_{2}$ y $\mathrm{O}_{3}$. Por su parte, diversos autores han encontrado evidencia sobre la naturaleza caótica de series temporales de PM (Chelani y Devotta 2006, Kumar et al. 2008, Lee y Lin 2008). Aún más, Salini y Pérez (2015) demostraron que $\mathrm{PM}_{2.5}$ recolectados en cuatro estaciones de monitoreo en Santiago, Chile, presentaron claros indicios de caoticidad. Pero, a pesar de esto, es posible el pronóstico a corto plazo aún en presencia de caos.

Este estudio tiene como objetivos caracterizar al contaminante $\mathrm{PM}_{10}$ originado en la ciudad de Cochabamba, mostrar su carácter no lineal e investigar su posible caoticidad. A su vez, presentar la situación actual de este contaminante, especialmente en época invernal y ser una contribución para llevar a cabo estudios más detallados.

\section{MATERIALES Y MÉTODOS}

\section{Área de estudio}

La ciudad de Cochabamba se encuentra entre los $17^{\circ} 23^{\prime}$ de latitud sur y los $66^{\circ} 09^{\prime}$ de longitud oeste del Meridiano de Greenwich, estando situada en el centro del país (Fig. 1). Es surcada por el ramal oriental de la Cordillera de los Andes, localizada en el valle del mismo nombre, siendo capital del Departamento de Cochabamba y de la provincia de Cercado. Posee una población de 616021 hab (INE 2012), en una superficie de aproximadamente $55631 \mathrm{~km}^{2}$. Cochabamba se sitúa en un valle interandino, esencialmente montañoso, situada a una altura de 2558 msnm. Cuenta con varios valles situados a diferentes alturas sobre el nivel del mar, siendo los más importantes Cochabamba, Sacaba, el Valle Alto y el de Cliza.

Cochabamba posee clima seco y templado. Las temperaturas en invierno no bajan más de $10{ }^{\circ} \mathrm{C}$ y en verano no suben más de $30^{\circ} \mathrm{C}$. La precipitación promedio anual es de $390 \mathrm{~mm}$; su humedad relativa es de $49 \%$, su presión atmosférica es 0.74 atm y su temperatura promedio anual es de $18{ }^{\circ} \mathrm{C}$ (Red MoniCA 2010). Red MoniCA cuenta con una estación meteorológica situada en la estación SEMAPA, en la zona norte de la ciudad (Fig. 2), donde se miden temperatura, pluviosidad, dirección y velocidad del viento, y radiación solar. Según mediciones de esta estación, la velocidad del viento promedio anual es de $3.9 \mathrm{~m} / \mathrm{s}$ siendo estable prácticamente todo el año, produciéndose leve aumento entre agosto y septiembre, meses en que se registran los vientos más fuertes. La mayor de las veces su dirección es del sur, del este y del oeste, lo que explicaría las elevadas concentraciones de ozono en la zona norte de la ciudad, 


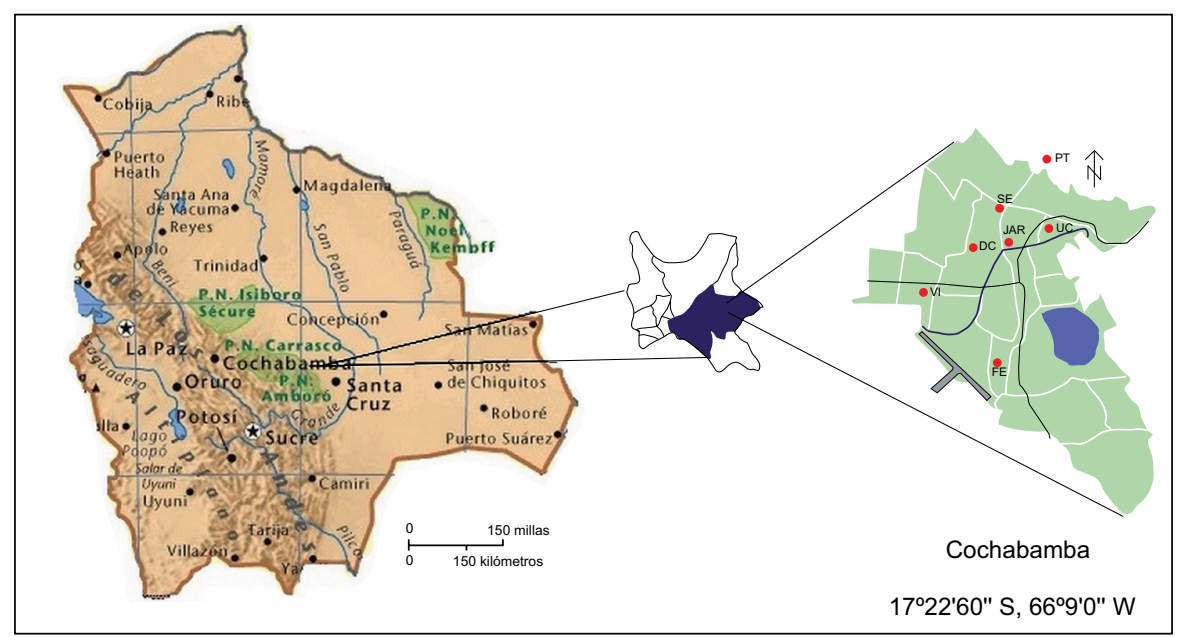

Fig. 1. Mapa de la ciudad de Cercado, Cochabamba, Bolivia

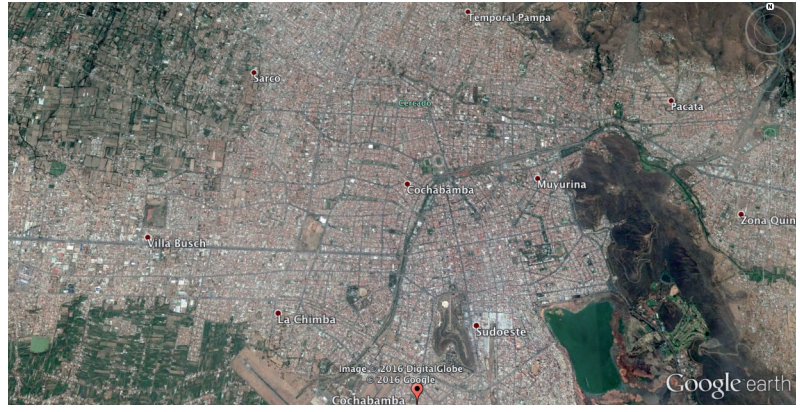

Fig. 2. Plano de las estaciones de monitoreo en la ciudad de Cercado, Cochabamba, Bolivia repitiéndose dicho patrón durante todo el año (Vargas y Luján 2006). Por su parte, la Administración de Aeropuertos y Servicios Auxiliares a la Navegación Aérea (ASANA), dispone de información meteorológica como velocidad del viento, temperatura, presión y humedad relativa (Fig. 3).

\section{Los datos}

Estos se obtuvieron desde la Red MoniCa. Cochabamba cuenta con tres estaciones de monitoreo de contaminación ambiental, que forman parte de esta Red (Fig. 2) y que realiza monitoreo con tecnología implementada en tres centros situados en la zona de
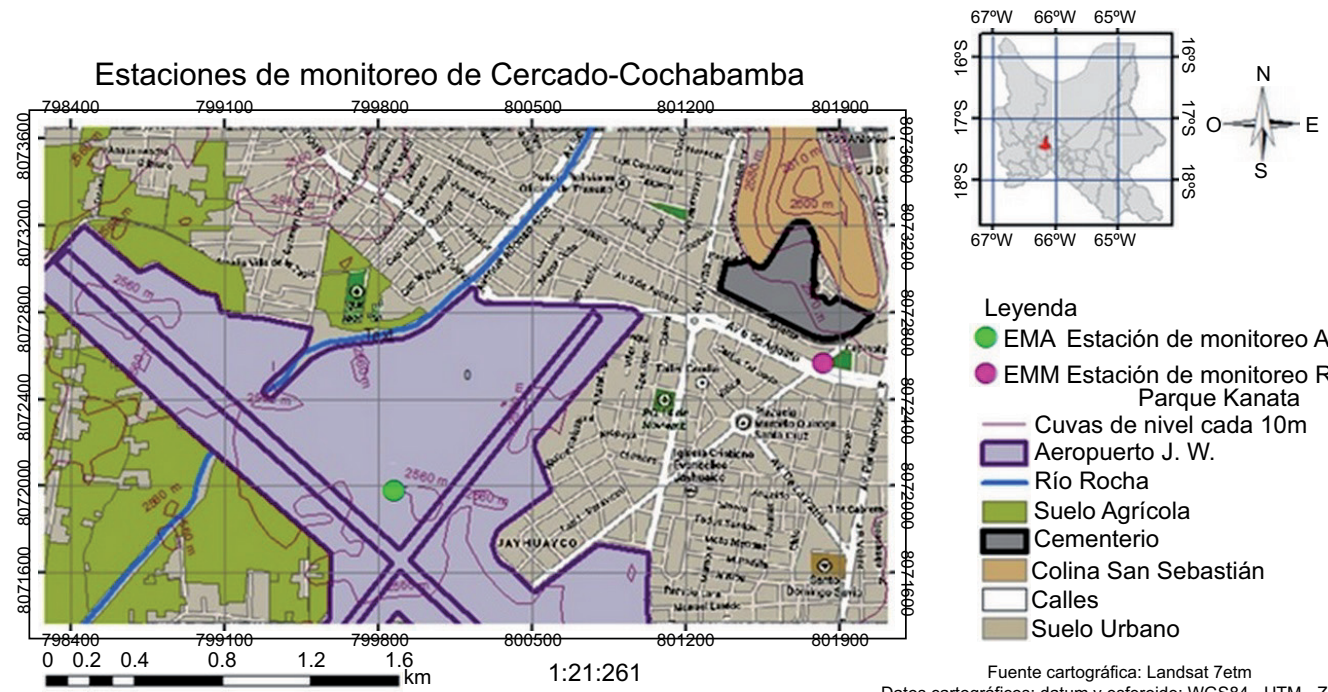

Leyenda

EMA Estación de monitoreo ASANA (EMA)

- EMM Estación de monitoreo RedMónica Parque Kanata

- Cuvas de nivel cada $10 \mathrm{~m}$

$\square$ Aeropuerto J. W.

— Río Rocha

Suelo Agrícola

$\square$ Cementerio

Colina San Sebastián

$\square$ Calles

Suelo Urbano

Fuente cartográfica: Landsat $7 \mathrm{etm}$

Datos cartográficos: datum y esferoide: WGS84 - UTM - Z19S Elaboración propia

Fig. 3. Diagrama esquemático de las estaciones de monitoreo de la ciudad de Cercado, Cochabamba, Bolivia. ASANA = Aeropuertos y Servicios Auxiliares a la Navegación Aérea, RedMonica: red de monitoreo de contaminación ambiental 
Semapa, Kanata (Fig. 3) y Coña Coña (en implementación). La estación Parque Kanata (PK) mide concentración de contaminantes como $\mathrm{O}_{3}, \mathrm{CO}, \mathrm{NOx}$, $\mathrm{SO}_{2}, \mathrm{PM}_{10}$ y variables meteorológicas con el fin de evaluar y comparar con los niveles establecidos en la ley del Medio Ambiente (Vargas y Luján 2006). A partir del año 2009 se comenzó a recopilar información desde el 3 de marzo a las 17:00 h. Originalmente, los datos son recolectados cada $30 \mathrm{~min}$, pero en este estudio se utiliza promedio horario. Es decir se tendrían 8760 datos para un año normal. En el caso de ser año bisiesto, habrá 8784 datos (año 2012). En general, la base de datos se recopila y procesa desde el sitio de internet que mantiene esta Red. En este estudio se usaron datos de $\mathrm{PM}_{10}$ recolectados desde la estación de monitoreo PK situada en la ciudad de Cercado, Cochabamba y con los que se realizaron análisis estadístico de ellos.

En este estudio se ha decidido emplear metodología de relleno lineal (Yang et al. 2005) debido, por una parte, a que todas las series (excepto el año 2013) presentan un bajo porcentaje de datos faltante (menor al $5 \%$ ) y por otra parte para evitar introducir efectos no lineales adicionales al sistema.

Uno de los métodos más usados en estadística para la detección de valores atípicos -outliers- involucra el concepto de cuartil e intercuartil (Chandler y Scott 2011) para un conjunto dado de datos. Así, para la detección de valores atípicos extremos se empleó la expresión

$\operatorname{LimSup}=\mathrm{Q} 3+3 \times(\mathrm{Q} 3-\mathrm{Q} 1)$

En consecuencia, valores mayores a LimSup se consideran valores atípicos.

\section{Herramientas adicionales en series temporales no lineales}

Además de las herramientas mencionadas en la Introducción, se han empleado otros parámetros estadísticos como la dimensión de correlación, entropía $\mathrm{K}$ de la información (Kolmogorov 1959), exponente de Hurst $(\mathrm{H})$ y exponente de Lyapunov $\left(\lambda_{\mathrm{L}}\right)$, que permiten indagar el grado de caoticidad que presenta una serie temporal (Grassberger y Procaccia 1983). La dimensión de correlación es una medida de la dimensión del espacio de fases. Si resulta entera es indicador de regularidad y si es fractal (no entera) es indicio de caos. Una dimensión de correlación más grande que cinco implica que la serie de tiempo presenta datos correspondientes a un proceso aleatorio (Sprott 2003). El exponente de Hurst (H) es una medida del grado al cual los datos pueden representarse mediante un camino aleatorio cuya pendiente corresponde al exponente de Hurst. Para movimiento Browniano ordinario el exponente es 0.5 . Valores entre 0.5 y 1.0 indican comportamiento determinista. Exponente de Hurst significativamente menor que 0.5 corresponde a comportamiento determinista y persistencia (Kantz y Schreiber 2004). El exponente más grande de Lyapunov se emplea en la detección de posible comportamiento caótico en una serie temporal (Eckmann et al. 1986). Si el máximo exponente de Lyapunov es negativo la serie corresponde a un sistema disipativo con un punto fijo estable. Si, en cambio, el movimiento se asienta sobre un ciclo límite, el máximo exponente de Lyapunov será cero. Si un sistema predominantemente determinista es afectado por ruido aleatorio, el máximo exponente de Lyapunov es infinito (Kantz y Schreiber 2004). Finalmente, si es positivo y finito es claro indicio de presencia de caos en la serie temporal. Para esta serie, la suma de todos los exponentes positivos de Lyapunov define la entropía de Kolmogorov, K (Kolmogorov 1959), siendo su recíproco el tiempo promedio de predictibilidad, $T_{p}=1 / K$. En Salini y Pérez (2015) se muestra cómo se determinan los exponentes de Lyapunov para concentraciones de $\mathrm{PM}_{2.5}$. En general, el exponente positivo más grande de Lyapunov de un sistema, $\lambda_{\mathrm{L}}$, se obtiene a partir de (Liu et al. 2004)

$T_{p} \leq \frac{1}{\lambda_{L}}$

\section{RESULTADOS}

El cuadro I presenta la estadística descriptiva para cada serie registrada en la estación de monitoreo PK entre 2009 y 2013 para $\mathrm{PM}_{10}$ en Cochabamba. Para todos los años en estudio el máximo valor de $\mathrm{PM}_{10}$ está por sobre la norma boliviana y de la OMS. Como es sabido, si una serie temporal presenta poca dispersión en sus datos, es señal inequívoca de homogeneidad en ellos. Sin embargo, en el cuadro I no se aprecia esto. El valor positivo del sesgo (o asimetría) que se muestra en este mismo cuadro presenta distribución lognormal con sesgos a la derecha. El cuadro II muestra los valores atípicos para $\mathrm{PM}_{10}$, tanto para época estival como invernal. Para época estival de cada año se muestra el valor límite superior y entre paréntesis están todos los valores que sobrepasan dicho umbral, siendo los tres datos que le preceden los últimos más altos de ese año. Lo mismo se muestra en la columna contigua para época invernal. 
CUADRO I. ESTADÍSTICA DESCRIPTIVA DE MATERIAL PARTICULADO PM 10 (HORARIA Y ANUAL PARQUE KANATA, COCHABAMBA, BOLIVIA)

\begin{tabular}{|c|c|c|c|c|c|c|c|c|}
\hline $\begin{array}{l}\mathrm{PM}_{10} \\
\text { año }\end{array}$ & $\begin{array}{l}\text { Número } \\
\text { de datos }\end{array}$ & $\begin{array}{l}\text { Número } \\
\text { real de datos }\end{array}$ & $\begin{array}{l}\text { Media } \\
\left(\mu \mathrm{g} / \mathrm{m}^{3}\right)\end{array}$ & $\begin{array}{c}\text { Máximo } \\
\text { valor }\left(\mu \mathrm{g} / \mathrm{m}^{3}\right)\end{array}$ & $\begin{array}{c}\text { Mínimo } \\
\text { valor }\left(\mu \mathrm{g} / \mathrm{m}^{3}\right)\end{array}$ & $\begin{array}{c}\text { Desviación } \\
\text { estándar }\left(\mu \mathrm{g} / \mathrm{m}^{3}\right)\end{array}$ & $\begin{array}{c}\text { Coeficiente } \\
\text { asimetría }\left(\mu \mathrm{g} / \mathrm{m}^{3}\right)\end{array}$ & $\begin{array}{l}\text { Curtosis } \\
\left(\mu \mathrm{g} / \mathrm{m}^{3}\right)\end{array}$ \\
\hline 2009 & 7279 & 7191 & 103.1 & 588 & 2 & 84.1 & 1.61 & 2.86 \\
\hline 2010 & 8760 & 8500 & 76.0 & 620 & 3 & 64.7 & 2.17 & 6.94 \\
\hline 2011 & 8760 & 8493 & 70.1 & 755 & 2 & 57.7 & 2.44 & 9.91 \\
\hline 2012 & 8784 & 8402 & 78.9 & 597 & 1 & 108.8 & 1.77 & 3.65 \\
\hline 2013 & 8760 & 7195 & 61.7 & 319 & 3 & 44.0 & 1.74 & 3.85 \\
\hline
\end{tabular}

CUADRO II. VALORES ATÍPICOS DE MATERIAL PARTICULADO PM PM $_{10}$ (HORARIA PERIODO ESTIVAL E INVERNAL PARQUE KANATA, COCHABAMBA, BOLIVIA)

\begin{tabular}{ccc}
\hline Año & Estival & Invernal \\
& LimSup $\left(\mu \mathrm{g} / \mathrm{m}^{3}\right)$ & LimSup $\left(\mu \mathrm{g} / \mathrm{m}^{3}\right)$ \\
\hline 2010 & $181(101)$ & $431(16)$ \\
& 284 & 588 \\
& 288 & 603 \\
& 411 & 620 \\
\hline 2011 & $173(99)$ & $370(19)$ \\
& 429 & 510 \\
& 462 & 520 \\
& 755 & 601 \\
\hline 2012 & $208(38)$ & $432(4)$ \\
& 316 & 443 \\
& 338 & 558 \\
& 376 & 597 \\
\hline
\end{tabular}

El cuadro III resume la cantidad de datos faltante para $\mathrm{PM}_{10}$ en la estación de monitoreo PK entre los años 2009 y 2013. El cuadro IV resume la estadística básica de $\mathrm{PM}_{10}$ horario pero para el periodo otoño-invierno. Mientras que el cuadro $\mathbf{V}$ resume la estadística de datos faltantes para el mismo periodo, en los cinco años de recopilación. La figura 4 muestra el promedio horario de concentraciones de $\mathrm{PM}_{10}$ (datos crudos) para la estación de monitoreo PK del año 2011. La figura 5 muestra el promedio horario anual de concentraciones de $\mathrm{PM}_{10}$ original para la estación de monitoreo PK del año 2012. Los datos faltantes se han rellenado con la metodología estándar de tipo lineal (representado por el símbolo '*' mostrado en la figura 6 empleando para ello una porción de la serie (año 2011, estación PK).

La figura 7 presenta el promedio de $\mathrm{PM}_{10}$ para cada día de la semana correspondiente al periodo de otoño-invierno del año 2012. La figura 8 muestra la

CUADRO III. ESTADÍSTICA DESCRIPTIVA DATOS FALTANTES DE MATERIAL PARTICULADO PM 10 (HORARIAY ANUAL PARQUE KANATA, COCHABAMBA, BOLIVIA)

\begin{tabular}{lcccccccc}
\hline $\begin{array}{l}\mathrm{PM}_{10} \\
\text { año }\end{array}$ & $\begin{array}{c}\text { Número } \\
\text { de datos }\end{array}$ & $\begin{array}{c}\text { Número } \\
\text { real de } \\
\text { datos }\end{array}$ & $\begin{array}{c}\text { Datos } \\
\text { faltante } \\
(\%)\end{array}$ & $\begin{array}{c}\text { Porcentaje } \\
\text { real \% }\end{array}$ & $\begin{array}{c}\text { Faltante } \\
\text { primer } \\
\text { mayor }\end{array}$ & $\begin{array}{c}\text { Faltante } \\
\text { primer mayor } \\
(\%)\end{array}$ & $\begin{array}{c}\text { Faltante } \\
\text { segundo } \\
\text { mayor }\end{array}$ & $\begin{array}{c}\text { Faltante } \\
\text { segundo mayor } \\
(\%)\end{array}$ \\
\hline 2009 & 7279 & 7191 & 88 & 1.2 & 24 & 27.3 & 17 & 19.3 \\
2010 & 8760 & 8500 & 260 & 3.1 & 86 & 33.1 & 36 & 13.8 \\
2011 & 8760 & 8493 & 267 & 3.1 & 74 & 27.7 & 30 & 1.2 \\
2012 & 8784 & 8402 & 382 & 4.5 & 113 & 29.6 & 108 & 28.3 \\
2013 & 8760 & 7195 & 1565 & 21.8 & 477 & 30.5 & 216 & 13.8 \\
\hline
\end{tabular}

CUADRO IV. ESTADÍSTICA DESCRIPTIVA DE MATERIAL PARTICULADO PM 10 (HORARIA PERIODO INVIERNAL PARQUE KANATA, COCHABAMBA, BOLIVIA)

\begin{tabular}{lcccccccc}
\hline $\begin{array}{l}\mathrm{PM}_{10} \\
\text { invierno }\end{array}$ & $\begin{array}{c}\text { Número } \\
\text { de datos }\end{array}$ & $\begin{array}{c}\text { Número } \\
\text { real de datos }\end{array}$ & $\begin{array}{c}\text { Media } \\
\left(\mu \mathrm{g} / \mathrm{m}^{3}\right)\end{array}$ & $\begin{array}{c}\text { Máximo } \\
\text { valor } \\
\left(\mu \mathrm{g} / \mathrm{m}^{3}\right)\end{array}$ & $\begin{array}{c}\text { Mínimo } \\
\text { valor } \\
\left(\mu \mathrm{g} / \mathrm{m}^{3}\right)\end{array}$ & $\begin{array}{c}\text { Desviación } \\
\text { normal } \\
\left(\mu \mathrm{g} / \mathrm{m}^{3}\right)\end{array}$ & $\begin{array}{c}\text { Coeficiente } \\
\text { asimetría } \\
\left(\mu \mathrm{g} / \mathrm{m}^{3}\right)\end{array}$ & $\begin{array}{c}\text { Curtosis } \\
\left(\mu \mathrm{g} / \mathrm{m}^{3}\right)\end{array}$ \\
\hline 2009 & 3672 & 3658 & 134.4 & 588 & 2 & 94.7 & 1.17 & 1.20 \\
2010 & 3672 & 3553 & 106.1 & 620 & 7 & 79.1 & 1.59 & 3.75 \\
2011 & 3672 & 3619 & 90.8 & 601 & 4 & 68.8 & 1.76 & 4.45 \\
2012 & 3672 & 3646 & 103.0 & 597 & 4 & 70.7 & 1.22 & 1.78 \\
2013 & 3672 & 3462 & 102.3 & 545 & 3 & 74.5 & 1.44 & 2.75 \\
\hline
\end{tabular}


CUADRO V. ESTADÍSTICA DESCRIPTIVA DATOS FALTANTES DE MATERIAL PARTICULADO PM 10 (HORARIA PERIODO INVIERNAL PARQUE KANATA, COCHABAMBA, BOLIVIA)

\begin{tabular}{cccccccrc}
\hline $\begin{array}{c}\text { PM }_{10} \\
\text { invierno }\end{array}$ & $\begin{array}{c}\text { Número } \\
\text { de datos }\end{array}$ & $\begin{array}{c}\text { Número } \\
\text { real de datos }\end{array}$ & $\begin{array}{c}\text { Total } \\
\text { faltantes }\end{array}$ & $\%$ & $\begin{array}{c}\text { Primer } \\
\text { mayor }\end{array}$ & $\begin{array}{c}\text { Primer } \\
\text { mayor }(\%)\end{array}$ & $\begin{array}{c}\text { Segundo } \\
\text { mayor }\end{array}$ & $\begin{array}{c}\text { Segundo } \\
\text { mayor }(\%)\end{array}$ \\
\hline 2009 & 3672 & 3658 & 14 & 0.4 & 4 & 28.9 & 4 & 28.9 \\
2010 & 3672 & 3553 & 119 & 3.3 & 86 & 72.3 & 13 & 10.9 \\
2011 & 3672 & 3619 & 53 & 1.5 & 30 & 56.6 & 10 & 18.9 \\
2012 & 3672 & 3646 & 26 & 0.7 & 7 & 26.9 & 4 & 15.4 \\
2013 & 3672 & 3462 & 210 & 5.8 & 57 & 27.1 & 51 & 24.3 \\
\hline
\end{tabular}

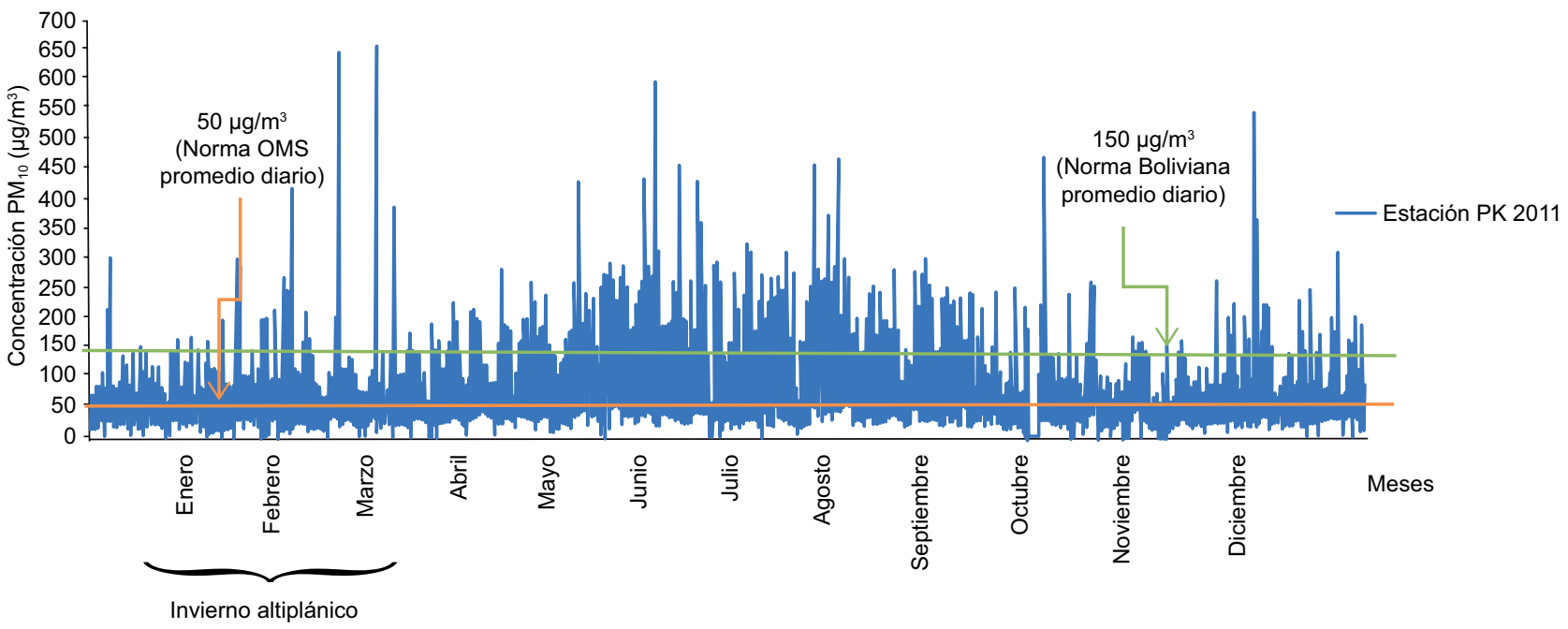

Fig. 4. Serie temporal horaria de material particulado $\mathrm{PM}_{10}$ del año 2011, para la estación de monitoreo Parque Kanata (PK), Cercado, Cochabamba, Bolivia

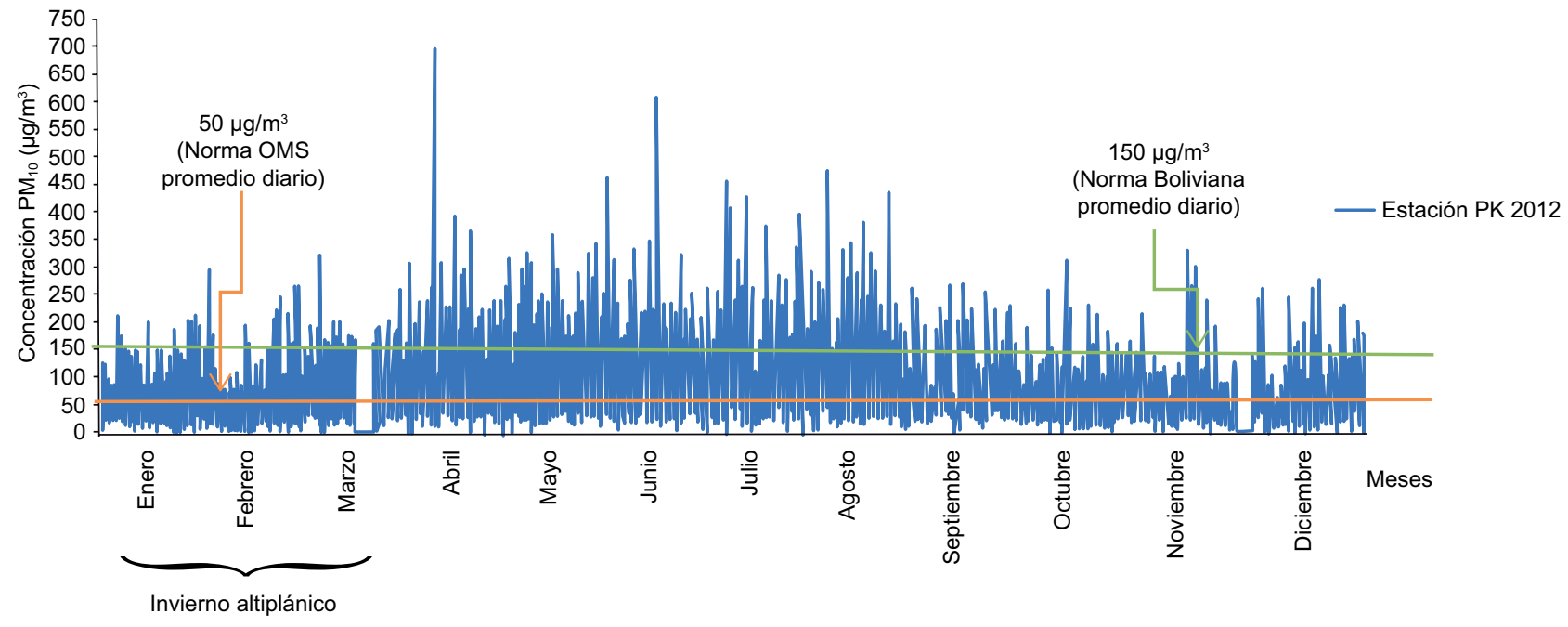

Fig. 5. Serie temporal horaria de material particulado $\mathrm{PM}_{10}$ año 2012, estación de monitoreo Parque Kanata (PK), Cercado, Cochabamba, Bolivia 


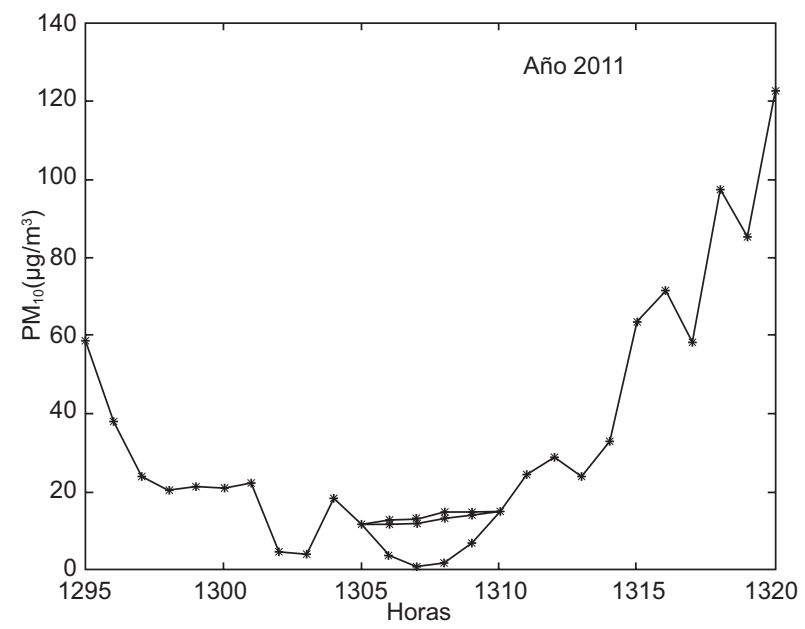

Fig. 6. Serie temporal de material particulado $\mathrm{PM}_{10}$ interpolada al año 2011, estación de monitoreo Parque Kanata (PK), Cercado, Cochabamba, Bolivia

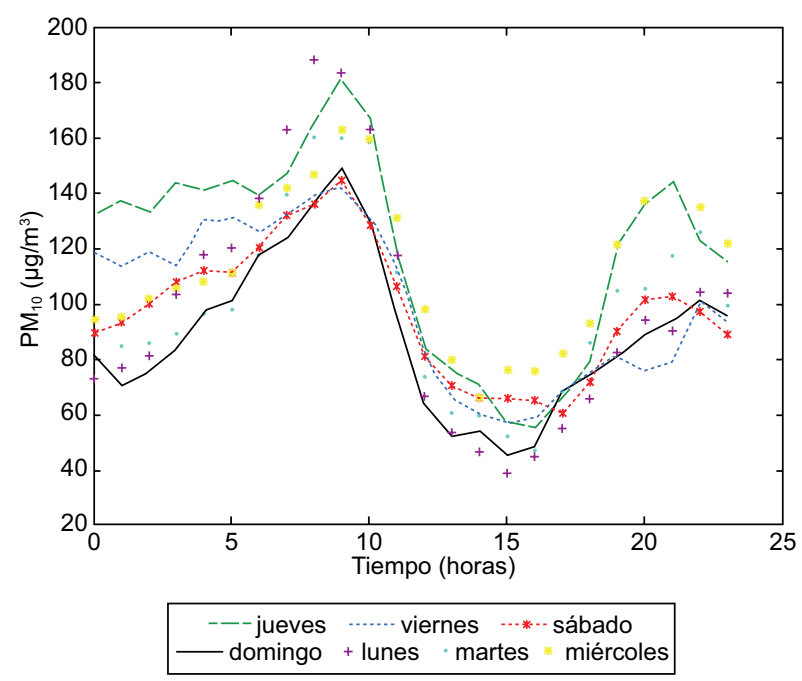

Fig. 7. Promedio diario de material particulado $\mathrm{PM}_{10}$ estación Parque Kanata (PK), Cochabamba, Bolivia, en el periodo invernal (otoño-invierno) 2011

función AMI para datos del año 2011, estación PK. Los gráficos para los demás años son bastante similares y sus valores se muestran en el cuadro VI. Por su parte, la figura 9 bosqueja la función FNN para el año 2011 (estación PK). Nuevamente, los otros años en estudio muestran resultados similares y sus valores se resumen en el cuadro VI. En este cuadro se presentan los valores de dos parámetros (tiempo de rezago y dimensión de encajamiento) calculados para efectuar el estudio de posible no linealidad para las series temporales de $\mathrm{PM}_{10}$ en la estación de monitoreo PK. Para todos los años en estudio el tiempo de rezago t, resultó ser 8 , y la dimensión de encaje, $d_{e}$,

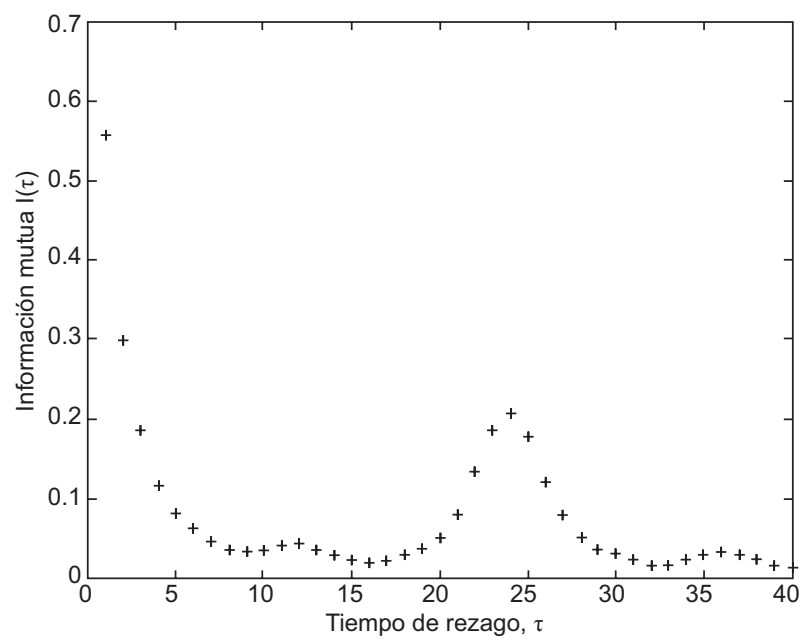

Fig. 8. Información binaria $\mathrm{I}(\tau)$ o mutua promedio (AMI, por sus siglas en inglés) de material particulado $\mathrm{PM}_{10}$ para el año 2011, serie horaria, estación Parque Kanata (PK)

CUADRO VI. PARÁMETROS PARA ESTUDIO DE NO LINEALIDAD EN MATERIAL PARTICULADO PM ${ }_{10}$ (HORARIA Y ANUAL PARQUE KANATA, COCHABAMBA, BOLIVIA)

\begin{tabular}{lcccc}
\hline $\begin{array}{l}\mathrm{PM}_{10} \\
\text { año }\end{array}$ & $\begin{array}{c}\text { Número } \\
\text { de datos }\end{array}$ & $\begin{array}{c}\text { Número } \\
\text { real de datos }\end{array}$ & $\begin{array}{c}\text { Tiempo } \\
\text { de rezago }\end{array}$ & $\begin{array}{c}\text { Dimensión } \\
\text { de encaje }\end{array}$ \\
\hline 2009 & 7279 & 7191 & 8 & 5 \\
2010 & 8760 & 8500 & 8 & 5 \\
2011 & 8760 & 8493 & 8 & 5 \\
2012 & 8784 & 8402 & 8 & 5 \\
2013 & 8760 & 7195 & 7 & 5 \\
\hline
\end{tabular}

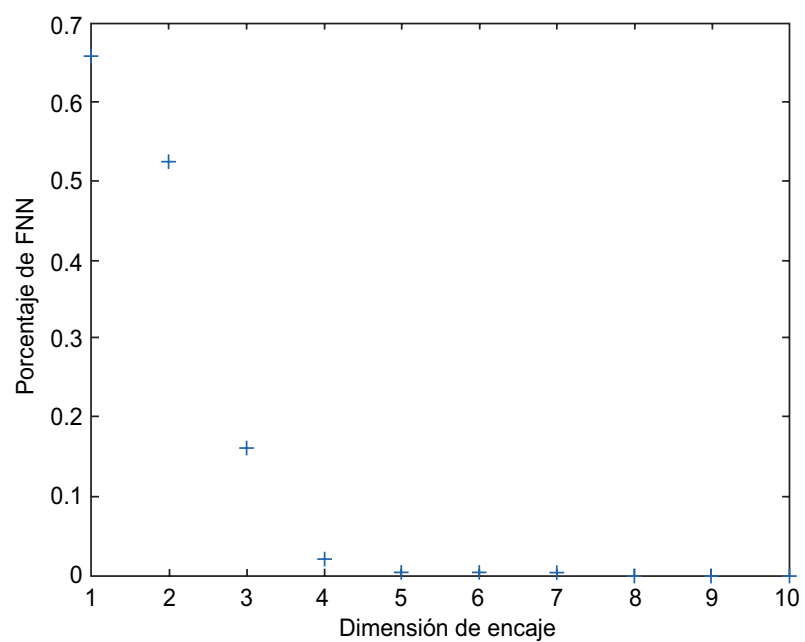

Fig. 9. Función falsos vecinos más cercanos (FNN, por sus siglas en inglés), de material particulado $\mathrm{PM}_{10}$ para el año 2011, serie horaria, estación Parque Kanata (PK) 
tuvo un valor de 5. La figura $\mathbf{1 0}$ muestra el logaritmo de la distancia como una función del intervalo de tiempo para diferentes puntos iniciales del año 2012 desde el 1 de abril hasta el 31 de agosto. En el cuadro VII se resumen los valores para algunos parámetros no lineales de interés. Los exponentes de Lyapunov calculados varían entre 0.656 y 0.749 . Estos exponentes se determinaron usando la dimensión de encaje de $d_{e}=5$.

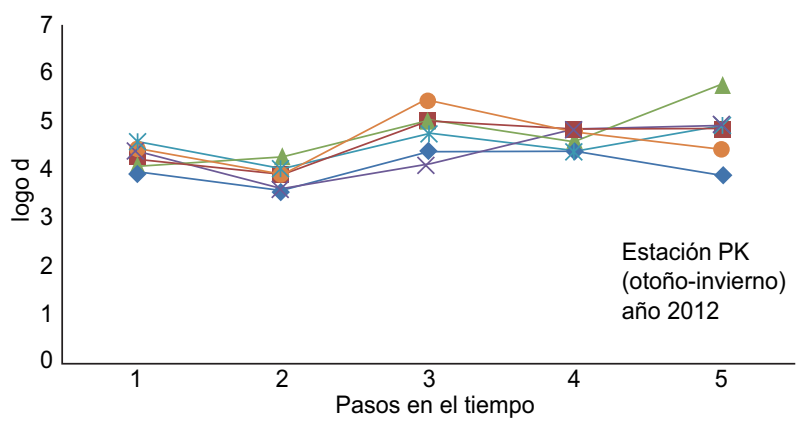

Fig. 10. Logaritmo de la distancia euclidiana $(\log d)$ entre vectores sucesivos 5-dimensional de material particulado $\mathrm{PM}_{10}$ horaria, estación Parque Kanata (PK), periodo otoño-invierno 2012

\section{DISCUSIÓN}

El cuadro II revela la presencia de datos anómalamente altos, indicando la presencia de valores de $\mathrm{PM}_{10}$ que se alejan bastante del promedio el cual es un valor inusualmente grande respecto de los datos. Estos valores, que no pueden atribuirse a un error y que revelan nueva información o tendencias que no habían sido predichas, por lo general, no se deberían ignorar. A su vez, revelan escasez de concentraciones elevadas de $\mathrm{PM}_{10}$ en toda la serie temporal. Para la buena gestión de la calidad del aire en una ciudad, son estos los valores que interesan desde el punto de vista operacional, puesto que presenta inconveniente en su construcción debido a que los parámetros del modelo se calculan a partir de valores históricos, y una escasa representación de alta concentración implicará, en general, una tendencia a no predecir valores extremos. Además, estos valores son muy difíciles de pronosticar por los modelos predictivos actuales tales como los deterministas así como lo estadísticos debido, por una parte a que tienden a promediar los valores y por otra, a la no linealidad y caoticidad que presentan dichos datos. En Pérez y Salini (2008) se presenta un nuevo modelo de pronóstico que consideraría estos valores extremos. Aún más, Salini y Pérez (2015) demostraron que al considerar la estacionalidad de las series temporales (casos anual y estacional) en la determinación del exponente de Lyapunov más positivo- empleando para ello los parámetros $d_{e}$ y $\tau$-, la única diferencia con dichos exponentes es que se incrementan en el periodo invernal. Lo mismo que hemos observado aquí. Estos exponentes entregan información acerca de cuán sensibles a las condiciones iniciales son los datos.

En el año 2009 del cuadro III, hay 7279 datos-de un total de 8760 , debido al inicio tardío de recolección de datos-de los cuales 88 son datos faltantes, correspondiendo al $1.2 \%$ del total de datos reales de la serie temporal. En general, se sugiere que la cantidad de datos faltantes no sea mayor que el $10 \%$ de los datos reales de la serie, que en nuestro caso se cumple para todos los años (siendo menores al $5 \%$ ), de acuerdo al cuadro III, exceptuando el año 2013, que presenta un $21.2 \%$ y debiera considerarse revisar la recopilación de esos datos.

La figura 4 muestra los vacíos provocados por los datos faltantes. También puede apreciarse que los niveles de concentración de $\mathrm{PM}_{10}$ exceden la mayor parte del año la norma boliviana (línea verde horizontal), y la de la OMS-correspondiente a la línea roja horizontal. Finalmente, el periodo de invierno altiplánico (enero-marzo) también presenta niveles de $\mathrm{PM}_{10}$ por sobre la norma, tanto de la OMS como la boliviana, pero en menor cuantía que el periodo de invierno. En la figura 5 también se observan vacíos. Al igual que el caso anterior, el periodo de

CUADRO VII. PARÁMETROS PARA ESTUDIO DE CAOS EN MATERIAL PARTICULADO PM 10 (HORARIA PERIODO INVERNAL PARQUE KANATA, COCHABAMBA, BOLIVIA)

\begin{tabular}{lccccccc}
\hline $\begin{array}{l}\text { PM } \\
\text { invierno }\end{array}$ & $\begin{array}{c}\text { Exponente } \\
\text { Lyapunov }\end{array}$ & $\begin{array}{c}\text { Dimensión } \\
\text { de correlación }\end{array}$ & $\begin{array}{c}\text { Dimensión } \\
\text { de encaje }\end{array}$ & $\begin{array}{c}\text { Tiempo } \\
\text { rezago }\end{array}$ & $\begin{array}{c}\text { Exponente } \\
\text { de Hurst }\end{array}$ & $\begin{array}{c}\text { Entropía } \\
(1 / \mathrm{h})\end{array}$ & $\begin{array}{c}\text { Máxima } \\
\text { predicción (h) }\end{array}$ \\
\hline 2009 & 0.749 & 3.754 & $5-6$ & 4 & 0.229 & 0.511 & 1.957 \\
2010 & 0.616 & 4.128 & $6-7$ & 3 & 0.255 & 0.486 & 2.057 \\
2011 & 0.613 & 4.085 & $6-7$ & 4 & 0.227 & 0.429 & 2.331 \\
2012 & 0.656 & 3.935 & $6-7$ & 3 & 0.205 & 0.349 & 2.865 \\
\hline
\end{tabular}


invierno altiplánico (enero-marzo) también presenta niveles de $\mathrm{PM}_{10}$ por sobre la norma boliviana y de la OMS, pero en menor cuantía que el periodo de invierno. Para los otros años 2009, 2010 y 2013 los resultados son similares, observándose este mismo efecto estacional. De acuerdo a la figura 6, si la serie temporal presenta signos de no linealidad (representado por el símbolo '+') se debería intentar llevar a cabo el relleno de la serie temporal empleando metodologías distintas a la lineal, lo cual se hará en un futuro trabajo. Es frecuente encontrar datos faltantes en una serie temporal. Esto complica el análisis y estudio de los datos. Dicha situación puede deberse al muestreo insuficiente, error en las mediciones, fallas de los aparatos de medición, a la mantención de los mismos o al cambio del sitio de monitoreo, entre otras posibles causas. Cualquier metodología de relleno empleada debe tener en cuenta la naturaleza lineal o no lineal de la serie, así como el tamaño de los espacios vacíos en la serie para seleccionar el método más adecuado (Norazian et al. 2008), donde diferentes metodologías se emplean para su relleno (Little y Robin 2002, Junninen et al. 2004).

Salini y Pérez (2006) hallaron para el tiempo de rezago un valor de 7 y 8 , y para la dimensión de encaje encontraron un valor de 4 , que son similares a los obtenidos en este estudio. Por su parte, con estos parámetros se estudió la caoticidad en las series temporales de $\mathrm{PM}_{10}$, lo cual está completamente relacionado con la predictibilidad de la serie temporal, mostrada en el cuadro VII, donde se observa que la dimensión de correlación calculada varía entre 3.75 y 4.13, cantidades no enteras y menores que cinco, siendo indicio de comportamiento caótico y no aleatorio. Estos valores son consistentes con la suposición de comportamiento caótico para las series de $\mathrm{PM}_{10}$. El exponente de Hurst es, para todos los años, mucho menor que cinco, lo que indica comportamiento determinista y antipersistencia (predominancia de oscilaciones entre valores alto y bajos). Así, los valores de los parámetros calculados para la estación PK -y mostrado en el cuadro VII- confirman que las concentraciones de $\mathrm{PM}_{10}$ son caóticas. Además, dado los valores de los exponentes de Lyapunov más grande mostrado en el cuadro VII, se observa que las series de $\mathrm{PM}_{10}$ analizadas presentan caos. La entropía del sistema fluctuó entre 0.349 y $0.511 \mathrm{~h}$, lo que a su vez nos indica que la máxima predictibilidad está entre $1.957 \mathrm{~h}$ y $2.865 \mathrm{~h}$, respectivamente. A pesar de ello, es posible efectuar pronóstico de $\mathrm{PM}_{10}$ pero de corto plazo, aún en presencia de caos en las series temporales. Valores positivos del máximo exponente de Lyapunov también fueron observados en Salini y Pérez (2015) para la ciudad de Santiago de Chile, pero para $\mathrm{PM}_{2.5}$.

El hecho que en la figura 10 muchas de las curvas exhiben un incremento lineal puede ser visto como una verificación de la divergencia exponencial que a su vez revela presencia de caos.

Se sugiere habilitar la estación Coña Coña y registrar en forma horaria el contaminante $\mathrm{PM}_{2.5}$ en las tres estaciones de monitoreo de esta ciudad para contar con información más detallada y definir una nueva norma de calidad del aire para este contaminante, ya que se estaría superando la norma de $\mathrm{PM}_{2.5}$ propuesta por la OMS.

Es importante diseñar un programa de mejoramiento de la calidad del aire para esta ciudad a través de un plan de descontaminación ambiental. Además, el MMAyA debería contar con un modelo de pronóstico oficial de calidad del aire, para $\mathrm{PM}_{10}$ (si aún no ha sido implementado), para informar a la autoridad competente en los días de episodios críticos y tomar decisiones que involucre la protección de la salud de la población. Se sugiere realizar mantención de estaciones de monitoreo en época estival, para que los datos faltantes no involucre época invernal.

\section{CONCLUSIONES}

Esta investigación ha mostrado que el contaminante $\mathrm{PM}_{10}$ monitoreado en Cochabamba, ha estado por sobre el límite permitido. Todos los años en estudio se observaron superación de la norma de la OMS y gran parte del año, especialmente en época invernal, se superó la norma boliviana. Esto es preocupante debido a que existe riesgo de afectación a la salud de la población, la cual puede presentarse durante todo el año. Además, se hallaron valores atípicos tanto en el periodo estival como en el invernal. La mayor concentración de $\mathrm{PM}_{10}$ se produce alrededor de las 9:00 h.

La dimensión de correlación resultó ser no entera, siendo indicio de caoticidad. Además, el exponente de Hurst resultó ser menor de 5, indicando que la serie es determinista. Se ha demostrado que la serie de $\mathrm{PM}_{10}$ presenta características de no linealidad. Por su parte, valores de parámetros que indican la posible caoticidad y no linealidad para $\mathrm{PM}_{10}$, fueron de 8 y 5 . Los exponentes de Lyapunov resultaron ser positivos (entre 0.6 y 0.7 ), lo que también es indicio de caoticidad. Esto está respaldado por los otros parámetros mostrados en el cuadro VII. La máxima predictibilidad resultó ser de $3.0 \mathrm{~h}$, aproximadamente, lo que permitirá hacer un pronóstico a corto plazo, 
aún en presencia de caos. Nuestros resultados contribuyen a la literatura actual puesto que introducen un análisis más detallado de la naturaleza no lineal de concentraciones de $\mathrm{PM}_{10}$, que pueden presentar sensibilidad bajo condiciones iniciales.

\section{AGRADECIMIENTOS}

Se agradecen el apoyo de la Facultad de Ingeniería de la Universidad Católica de la Ssma. Concepción brindada a la Srta. Evelin Medina por cubrir parte de su estadía en el Depto. de Matemática y Física Aplicadas de dicha Universidad (entre el 8 y el 29 de agosto de 2012), así como al Departamento de Física de la Facultad de Ciencias y Tecnología de la Universidad Mayor de San Simón, Cochabamba. Este trabajo fue financiado, en parte, por el proyecto DIN 13/2016 (444713) de la Universidad Católica de la Ssma. Concepción.

\section{REFERENCIAS}

Abarbanel H. (1996). Analysis of observed chaotic data, Institute for Nonlinear Science, Springer-Verlag, Nueva York, EUA, 272 pp.

Bacopé-Moscoso D. (2003). La red de monitoreo de la calidad del aire de Cochabamba (Red MoniCA). ActaNova 2 (3), $10 \mathrm{pp}$.

Chandler R. y Scott M. (2011). Statistical methods for trend detection and analysis in the environmental sciences. John Wiley and Sons Ltd., West Sussex, Reino Unido, 388 pp.

Chelani A. B. y Devotta S. (2006). Nonlinear analysis and prediction of coarse particulate matter concentration in ambient air. J. Air Waste Manag. Assoc. 56 (1), 78-84. DOI: $10.1080 / 10473289.2006 .10464432$

Cifuentes L. A., Vega J., Köpfer K. y Lave L. B. (2000). Effect of the fine fraction of particulate matter versus the coarse mass and other pollutants on daily mortality in Santiago, Chile. J. Air Waste Manag. Assoc. 50 (8), 1287-1298.

Díaz V. y Páez C. (2006). Contaminación por material particulado en Quito y caracterización química de las muestras. Acta Nova-Quito, Ecuador 3 (2), 308-322.

Eckmann J. P., Oliffson S., Kamphorst S., Ruelle D. y Ciliberto C. (1986). Lyapunov exponents from time series. Phys. Rev. A 34 (6), 497-4979.

Fraser A. M. y Swinney H. L. (1986). Independent coordinates for strange attractors from mutual information. Phys. Rev. A 33 (2), 1134-1140.

DOI: 10.1103/PhysRevA.33.1134
Grassberger P. y Procaccia L. (1983). Characterization of strange attractors. Phys. Rev. Lett. 50 (5), 346-349. DOI: 10.1103/PhysRevLett.50.346

Ilabaca M., Olaeta I., Campos E., Villaire J., Telles-Rojo M. y Romieu, I. (1999). Association between levels of fine particulate and emergency visits for pneumonia and other respiratory illnesses among children in Santiago, Chile. J. Air Waste Manag. Assoc. 49 (Spec. No. 9), 154-163.

INE (2012). Características de población y viviendas. Censo Nacional de Población y Vivienda 2012, Instituto Nacional de Estadísticas. La Paz, Bolivia, $32 \mathrm{pp}$.

Junninen H., Niska H., Tuppurainen K., Ruuskanen J. y Kolehmainen M. (2004). Methods for imputation of missing values in air quality data sets. Atmos. Environ. 38 (18), 2895-2907.

DOI: 10.1016/j.atmosenv.2004.02.026

Kantz H. y Schreiber T. (2004). Nonlinear time series analysis. $2^{\mathrm{da}}$ ed. Cambridge University Press, Cambridge, Reino Unido, 387 pp.

Kolmogorov A. N. (1959). Entropy per unit time as a metric invariant of automorphisms. Dokl. Akad. Nauk SSSR 124, 754-755.

Kumar U., Prakash A. y Jain V.K. (2008). Characterization of chaos in air pollutants: A Volterra-Wiener-Korenberg series and numerical titration approach. Atmos. Environ. 42 (7), 1537-1551. DOI: $10.1016 /$ j.atmosenv.2007.10.076

Lee C. K. y Lin S. C. (2008). Chaos in air pollutant concentration (APC) time series. Aerosol Air Qual. Res. 8 (4), 381-391.

Little R. y Rubin B. (2002). Statistical analysis with missing data, $2^{\text {da }}$ ed. Wiley, Nueva York, EUA, 304 pp.

Liu J. Q., Zhang T. Q. y Yu S. K. (2004). Chaotic phenomenon and the maximum predictable time scale of observation series of urban hourly water consumption. J. Zhejiang Univ. Sci. 5 (9), 1053-1059. DOI: 10.1631 jzus.2004.1053

Lorenz E. N. (1963). Deterministic nonperiodic flow. J. Atm. Sci. 20, 130-141.

Melgarejo I., Soria-Sánchez R., Spielvogel H., Orozco D., Villanueva W. y Cajas N. (2010). Salud pulmonar y contaminación ambiental en comerciantes de las ciudades de La Paz y El Alto. BIOFARBO 18 (1), 42-53.

MMAyA (2010). Informe Nacional de la Calidad del Aire 2008-2009. Red MoniCA. Ministerio de Medio Ambiente y Agua. La Paz, Bolivia, 35 pp.

Molina M. J. y Molina L. T. (2004). Megacities and atmospheric pollution. J. Air Waste Manag. Assoc. 54 (6), 1047-3289.

DOI: $10.1080 / 10473289.2004 .10470936$ 
Morales R. G. E. (2006). Contaminación atmosférica urbana: episodios críticos de contaminación ambiental en la ciudad de Santiago. Editorial Universitaria, Santiago, Chile, 327 pp.

Norazian M. N., Shruki Y. A., Azam R. M. y Mustafa Al Bakri A. M. (2008). Estimation of missing values in air pollution data using single imputation techniques. ScienceAsia 34 (2008), 341-345.

DOI: $10.2306 /$ scienceasia1513-1874.2008.34.341

OMS (2006). Guías de calidad del aire de la OMS relativas al material particulado, el ozono, el dióxido de nitrógeno y el dióxido de azufre. Organización Mundial de la Salud, Ginebra, Suiza, 25 pp.

Pareja A., Hinojosa M. y Luján M. (2011). Inventario de emisiones atmosféricas contaminantes de la ciudad de Cochabamba, Bolivia, año 2008. Acta Nova 5 (3), 344-373.

Pérez P. y Reyes J. (2001). Prediction of particulate air pollution using neural techniques. Neural Comput. Appl. 10 (2), 165-171. DOI: 10.1007/s005210170008

Pérez P. y Salini G. (2008). PM $_{2.5}$ forecasting in a large city: Comparison of three methods. Atmos. Environ. 42 (35), 8219-8224.

DOI: 10.1016/j.atmosenv.2008.07.035

Peters A. y Dockery D.W. (2005). Lung biology in health and disease. En: Air pollutants and the respiratory tract. . (M. Foster y D.L. Costa, Eds.) CRC Press. Boca Raton, EUA, pp. 1-20.

Raabe O. G. (1999). Respiratory exposure to air pollutants. En: Air pollutants and the respiratory tract (D.L. Swift, W.M. Foster, Eds.). Nueva York, EUA, pp. 39-74.

Raga G.B. y Le Moyne L. (1996). On the nature of air pollution dynamics in Mexico city- I. Nonlinear analysis. Atmos. Environ. 30 (23), 3987-3993.

DOI: $10.1016 / 1352-2310(96) 00122-7$
Red MoniCA (2010). Informe nacional de la calidad del aire 2008-2009. Ministerio del Medio Ambiente y Agua. La Paz, Bolivia, 35 pp.

Reglamentación Ley del Medioambiente (1992). № 1333, Gaceta Oficial del Gobierno de Bolivia. Gaceta Oficial de Bolivia. 15 de junio 1992, 129 pp.

Salini G. y Pérez P. (2006). Estudio de series temporales de contaminación ambiental mediante técnicas de redes neuronales artificiales. Ingeniare 14 (3), 284-290. DOI: $10.4067 / \mathrm{S} 0718-33052006000200012$

Salini G. y Pérez P. (2015). A study of the dynamic behavior of fine particulate matter in Santiago, Chile. Aerosol Air Qual. Res. 15 (1), 154-165.

DOI: 10.4209/aaqr.2013.10.0323

Schwartz J., Laden F. y Zanobetti A. (2002). The concentration-response relation between $\mathrm{PM}_{2.5}$ and daily deaths. Environ. Health Perspec. 110 (10), 1025-1029.

Sivakumar B., Wallender W., Horwath W. y Mitchell J. (2007). Nonlinear deterministic analysis of air pollution dynamics in a rural and agricultural setting. Adv. Complex Syst. 10 (4), 581-597. DOI: $10.1142 / \mathrm{S} 0219525907001288$

Sprott J. C. (2003). Chaos and time-series analysis. Oxford University Press, Oxford, Reino Unido, 528 pp.

Takens F. (1981). Detecting Strange Attractors in Turbulence. En: Dynamical systems and turbulence, lecture notes in mathematics. (D.A.Rand y L.S. Young, Eds.). Springer-Verlag, Berlín, Alemania, pp. 366-381.

Vargas I. y Luján M. (2006). Memorias de la red MoniCA Cochabamba 2001-2006. Documento Universidad Católica Boliviana San Pablo, Cochabamba, Bolivia, $56 \mathrm{pp}$.

Yang W. Y., Cao W., Chung T-S. y Morris J. (2005). Applied numerical methods using Matlab, John Wiley \& Sons. Nueva Jersey, EUA, 511 pp. 